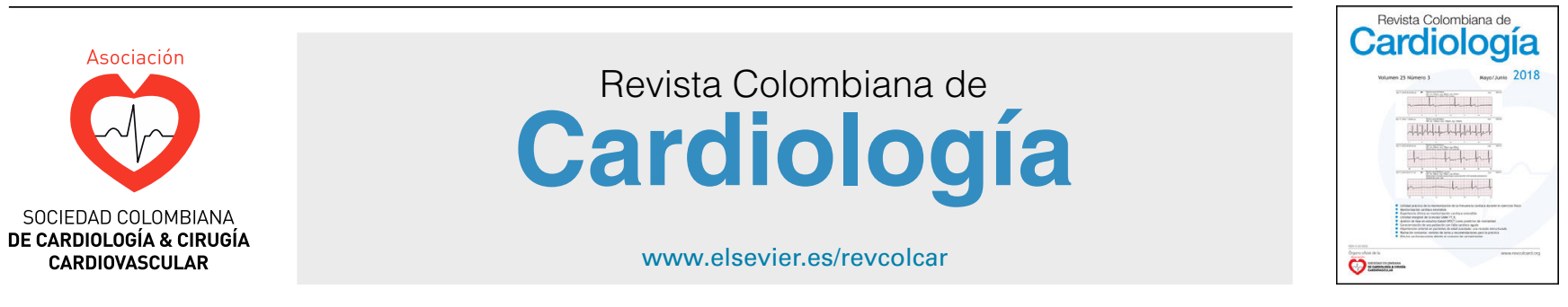

ARTÍCULO ESPECIAL

\title{
Riesgos osteomusculares: Patología ortopédica en el cardiólogo intervencionista
}

\author{
Mario Javier Vélez M. \\ Clínica Las Vegas, Medellín, Colombia \\ Recibido el 18 de septiembre de 2019; aceptado el 2 de enero de 2020
}

\author{
PALABRAS CLAVE \\ Dolor de espalda; \\ Enfermedades \\ profesionales; \\ Cardiólogo \\ intervencionista; \\ Equipo de protección \\ personal; \\ Protección \\ radiológica
}

\begin{abstract}
Resumen El dolor de espalda es uno de los síntomas más relacionados con los riesgos de salud ocupacional. Es de alta prevalencia en la población Norteamericana, y afecta principalmente a aquellas personas que, en su trabajo, deben usar la fuerza mecánica. Por consiguiente, es un riesgo laboral para profesionales de medicina intervencionista, como radiólogos, neurorradiólogos y cardiólogos intervencionistas. De hecho, en varios artículos de estudios observacionales este fenómeno se conoce como "enfermedad del disco intervencionista". Dicha condición está muy relacionada con el equipamiento de protección personal que suele ser de mayor peso, como los delantales de plomo usados para evitar la radiación ionizante, o con entornos de laboratorio de cateterización que promueven posturas ergonómicas inadecuadas. Se ha documentado que, en comparación con otros cirujanos, como los ortopedistas, estos profesionales de la medicina invasiva tienen mayor riesgo de presentar afecciones ortopédicas de la columna vertebral, aunque estos últimos tienen tiempos de procedimientos quirúrgicos más prolongados.
\end{abstract}

Por otra parte, la mayor tasa de incidencia de lesiones axiales en los cardiólogos intervencionistas parece estar relacionada con la combinación del uso de delantal de plomo y los procedimientos de cateterismo.

En estudios de prevalencia se ha demostrado que al comparar ortopedistas, reumatólogos y cardiólogos intervencionistas, estos últimos han presentado entre un 49,4 y un $53 \%$ de lesiones músculo-esqueléticas.

Los métodos para reducir la carga de peso se han centrado en innovaciones basadas en la fabricación de alternativas sin plomo para el blindaje; de igual manera, el uso de protectores móviles apoyados en el piso reduce sustancialmente la exposición de todo el cuerpo.

En cuanto a ergonomía, se requieren estudios de innovación con expertos en radiación, médicos y administradores a fin de dise nar una sala de radiología intervencionista adecuada.

( 2020 Sociedad Colombiana de Cardiología y Cirugía Cardiovascular. Publicado por Elsevier España, S.L.U. Este es un artículo Open Access bajo la licencia CC BY-NC-ND (http:// creativecommons.org/licenses/by-nc-nd/4.0/). 


\section{KEY WORDS}

Back pain; Occupational diseases; Interventional cardiologist; Personal protection equipment; Radiation protection

\section{Musculoskeletal risks: Orthopaedic disorders in the interventional cardiologist}

Abstract Back pain is one of the symptoms most associated with occupational health risks. It is highly prevalent in the North American population, and mainly affects those people that have to use mechanical effort in their work. It is, therefore, an occupational risk for interventionist medicine personnel, such as radiologists, neuro-radiologists and interventional cardiologists. As such, in several observational studies this phenomenon is known as "interventionist disc"'. This condition is closely associated with the personal radiation protection equipment that is usually very heavy. These include the leaded aprons used to protect against ionising radiation, or in catheterisation laboratories that promote inadequate ergonomic postures. There is evidence that, compared to other surgeons such as in orthopaedics, these invasive medicine professionals have a higher risk of spinal column problems, although these latter have longer surgical procedure times.

On the other hand, the higher incidence rate of axial injuries in interventional cardiologists seems to be associated with the combination of using a leaded apron and the catheterisation procedures.

It has been shown in prevalence studies that compare orthopaedists, rheumatologists, and interventionist cardiologists that these latter had between $49.4 \%$ and $53 \%$ musculoskeletal lesion.

The methods to reduce weight load have been focused on innovations based on the manufacture of lead-free alternatives for shielding. The use of mobile protectors supported on the floor also substantially reduces whole body exposure.

As regards ergonomics, new studies are required with experts in radiation, doctors and administrators, with the aim of designing an appropriate interventional radiology room.

(c) 2020 Sociedad Colombiana de Cardiología y Cirugía Cardiovascular. Published by Elsevier España, S.L.U. This is an open access article under the CC BY-NC-ND license (http:// creativecommons.org/licenses/by-nc-nd/4.0/).
El dolor de espalda es una afección que afecta entre el 50 y el $80 \%$ de los estadounidenses en algún momento de sus vidas ${ }^{1-6}$. Es común entre las personas cuyo trabajo incluye levantamiento, flexión y conducción extensa, ya que estos son factores de riesgo bien establecidos para su aparición ${ }^{1-9}$. Experiencia anecdótica entre radiólogos, especialmente aquellos involucrados en largos procedimientos angiográficos, intervencionistas y neurorradiológicos, sugiere que usar delantales de plomo podría ser un factor de riesgo para padecer dolor de espalda.

Los estudios observacionales indican el riesgo ocupacional involucrado en la práctica de la medicina intervencionista, particularmente respecto a los problemas ortopédicos ${ }^{10,11}$. Los autores han llamado la atención sobre un peligro ocupacional marcado, conocido como "enfermedad del disco intervencionista",12, y atribuyen estas lesiones ortopédicas a los efectos acumulativos del uso de delantales de plomo y entornos de laboratorio de cateterización mal diseñados, que promueven posturas ergonómicas incómodas (por ejemplo, monitores ubicados en posiciones de visión incómodas y no en la línea natural de la vista).

En la encuesta SCAI de 2004 casi la mitad de 424 encuestados informaron problemas con la columna vertebral ${ }^{13}$, una incidencia dramáticamente mayor que el 2,3\% de incidencia de enfermedades crónicas de la espalda en poblaciones estándar ${ }^{14}$. De aquellos que informaron problemas de columna vertebral, el 70\% estaba en el área lumbosacra y el $30 \%$ en el área cervical. Más de un tercio manifestó que sus problemas de columna vertebral les habían hecho perder el trabajo, y una cuarta parte informó problemas relacionados con sus caderas, rodillas o tobillos.

La encuesta también encontró una relación significativa entre la cantidad de años trabajados en el laboratorio de cateterización cardíaca y la incidencia de problemas de la columna vertebral. Los médicos que habían registrado 21 años o más en el laboratorio después de la especialización informaron acerca de una alarmante incidencia del $60 \%$ de problemas de columna vertebral en comparación con el $26 \%$ que trabajaban menos de 5 años después de completar la especialidad. Si bien estos datos podrían estar sujetos a un sesgo en el informe ya que los afectados por problemas ortopédicos tienen más probabilidades de responder a una encuesta de este tipo, otros datos de la encuesta recopilados de radiólogos sugieren que el problema es real ${ }^{10,11}$. Además, en comparación con los cirujanos ortopédicos, que ocasionalmente usan un delantal de plomo, y reumatólogos que no usan plomo, los cardiólogos invasivos tienen mayor incidencia de problemas de la columna vertebral ${ }^{12}$.

En una encuesta de 2012 aplicada en estadounidenses de 18 años y más, el $27 \%$ de los encuestados había experimentado dolor de espalda en los últimos tres meses ${ }^{15}$. La tasa de dolor de espalda fue mayor en edades avanzadas que en las cohortes más jóvenes. Las tasas de enfermedad de cuello y espalda aumentan en los médicos intervencionistas. Adicionalmente, la mayor frecuencia de días de trabajo perdidos por dolor de espalda y cuello se encontró en los médicos de cardiología intervencionista. Sin embargo, el 
aumento del dolor de cuello y espalda en dichos médicos no se correlacionó directamente con los tiempos operatorios o de procedimiento.

La cirugía ortopédica tuvo tiempos quirúrgicos más prolongados, pero los médicos de cardiología intervencionista tuvieron tasas más altas de dolor de cuello y espalda. Los médicos de cardiología intervencionista usan prendas de protección personal en la mayoría de los procedimientos, mientras que los cirujanos ortopedistas tienen menos procedimientos en los que usan prendas de plomo ${ }^{12}$. La mayor tasa de lesiones axiales en los médicos de cardiología intervencionista sugiere que la combinación de delantal de plomo y procedimientos dirigidos por catéter conduce a un diferente espectro y severidad de las lesiones.

Ross et al., ${ }^{12}$ acuñaron el término "enfermedad del disco intervencionista" cuando analizaron los resultados de su encuesta $(n=714)$ comparando a los cardiólogos intervencionistas (que realizan muchos procedimientos con delantales) con los cirujanos ortopédicos (que realizan muchos procedimientos, pero con menos delantales) y reumatólogos (que hacen pocos procedimientos). Los cardiólogos intervencionistas tuvieron más problemas en varias categorías, incluido el tratamiento para el dolor de espalda o cuello (53\%), y una incidencia mucho mayor de discos cervicales herniados $(6,5 \%)$. Significativamente más intervencionistas faltaron al trabajo debido a dolor de espalda o ciática, con un número significativamente mayor de días perdidos, mientras que 6 intervencionistas tuvieron una carrera corta o limitada; ninguno de los controles experimentó este contratiempo. Al comparar los que usan delantales y los que no lo hacen, los problemas similares fueron más frecuentes para los usuarios de delantales.

Una encuesta reciente de los miembros de la Sociedad de Angiografía e Intervenciones Cardiovasculares mostró que aproximadamente la mitad $(49,4 \%)$ de los encuestados tenía problemas músculo-esqueléticos que se asociaron significativamente con la cantidad de casos $(p<0,001)$ y la edad ( $p$ $<0,001)$. En cuanto a los encuestados, el $35 \%$ tenía dolor lumbar, el $25 \%$ dolor cervical y el $20 \%$ dolor en la cadera, las rodillas o los tobillos. Los periodos de ausencia relacionados con la salud se informaron en el $9 \%$ de los encuestados y en el $36 \%$ para aquellos entre 61 y 70 años de edad.

Una consideración bien reconocida para los médicos de cardiología intervencionista es la necesidad de protección contra la radiación. Aunque las prendas de plomo son extremadamente útiles en la protección contra la radiación, el uso de blindaje de plomo está asociado con mayores índices de lesiones. Tanto el peso inadecuado como el ajuste de las prendas de plomo pueden influir en la probabilidad de lesiones y dolor músculo-esquelético. Las prendas de plomo demasiado grandes pueden hacer que la dispersión pase a través de los grandes espacios en los brazos y haya mayor exposición a la radiación ${ }^{16}$.

Adicionalmente, casi la mitad de los usuarios de prendas de protección dijo que eran incómodas ${ }^{17}$. Las prendas de dos piezas, configuración de falda y chaleco, pueden reducir la presión de carga en la columna cervical y torácica; sin embargo, ningún estudio ha evaluado directamente las tasas de complicaciones ortopédicas según el diseño de la plataforma. Las prendas de protección de plomo enrolladas son más pesadas, pero pueden ofrecer menos tensión axial que las prendas de protección de frente solamente porque el peso se distribuye de manera uniforme.

Los delantales deben ser relativamente pesados para proteger adecuadamente a los intervencionistas ocupados, que se colocan en el lugar más caluroso de la habitación durante largos períodos de tiempo. Hasta que se realicen avances considerables en las pruebas y los informes obligatorios, la garantía de una protección segura se logra prácticamente al elegir prendas que contienen plomo con clasificaciones de equivalencia de $0,5 \mathrm{~mm} \mathrm{~Pb}$ o más, desde la línea axilar posterior hasta la línea axilar posterior opuesta y revisando cuidadosamente el etiquetado no revelado de doble grosor del panel. Los delantales livianos pueden ser satisfactorios para el personal auxiliar que circula y se aprovecha de la ley del cuadrado inverso o se queda frecuentemente detrás de una barrera o pared.

Diferentes estilos de delantal están disponibles para distintos propósitos y preferencias. Las categorías principales son la espalda cerrada frente a la abierta (según la presencia o ausencia de materiales atenuantes en una parte sustancial del cuerpo detrás de las líneas axilares posteriores), una pieza vs. dos piezas (por ejemplo delantal contra falda y chaleco) y con cinturón vs. sin cinturón de una sola pieza. En la actualidad es raro ver el estilo antiguo de abrigo de una pieza sin abrochar, con espalda cerrada, debido al enorme peso suspendido en los hombros. En un estudio, agregar un cinturón disminuyó sustancialmente la carga de la columna vertebral y se asoció con un dolor significativamente menor ${ }^{27}$. En el mismo estudio, una falda y un chaleco de dos piezas, que también alejan un poco de peso de la columna, no se asoció con un dolor significativamente diferente vs. una sola pieza con cinturón. Un gran estudio de encuesta no mostró una diferencia significativa en el dolor o la discapacidad entre prendas de una y dos piezas ${ }^{28}$, y se dispone de pocos datos. En general, los delantales con respaldo abierto son mucho más livianos que los modelos con respaldo cerrado que brindan una protección comparable, y están disponibles en modelos con cinturón para reducir el peso en la columna vertebral.

Los delantales cerrados pueden ser mejores para las personas que pueden dar la espalda al paciente durante la fluoroscopia, como técnicos de limpieza o enfermeras. Esto no es un problema para quien opera el pedal de fluoroscopia, por razones obvias, y la cantidad de dispersión secundaria desde las paredes de la habitación hasta la espalda del operador es insignificante. Una persona con dolor de columna que usa una prenda cerrada puede beneficiarse con un delantal de una sola pieza con cinturón, con una capacidad nominal de $0,5 \mathrm{~mm}$ Pb y que contiene plomo predominantemente. Tales modelos pueden ser los más ligeros entre los delantales totalmente protectores y también son generalmente de bajo costo. Las afirmaciones de que los delantales con peso adicional en la espalda reducen el dolor de la columna vertebral debido a una mejor distribución no se basan en la evidencia, pero un usuario con dolor de la columna vertebral puede querer probar todas las opciones para determinar el mejor estilo.

Las innovaciones recientes se han centrado en los métodos para reducir la carga de peso y, por lo tanto, en las lesiones del esqueleto axial asociadas con los procedimientos de carga. Por ejemplo, ahora los fabricantes 

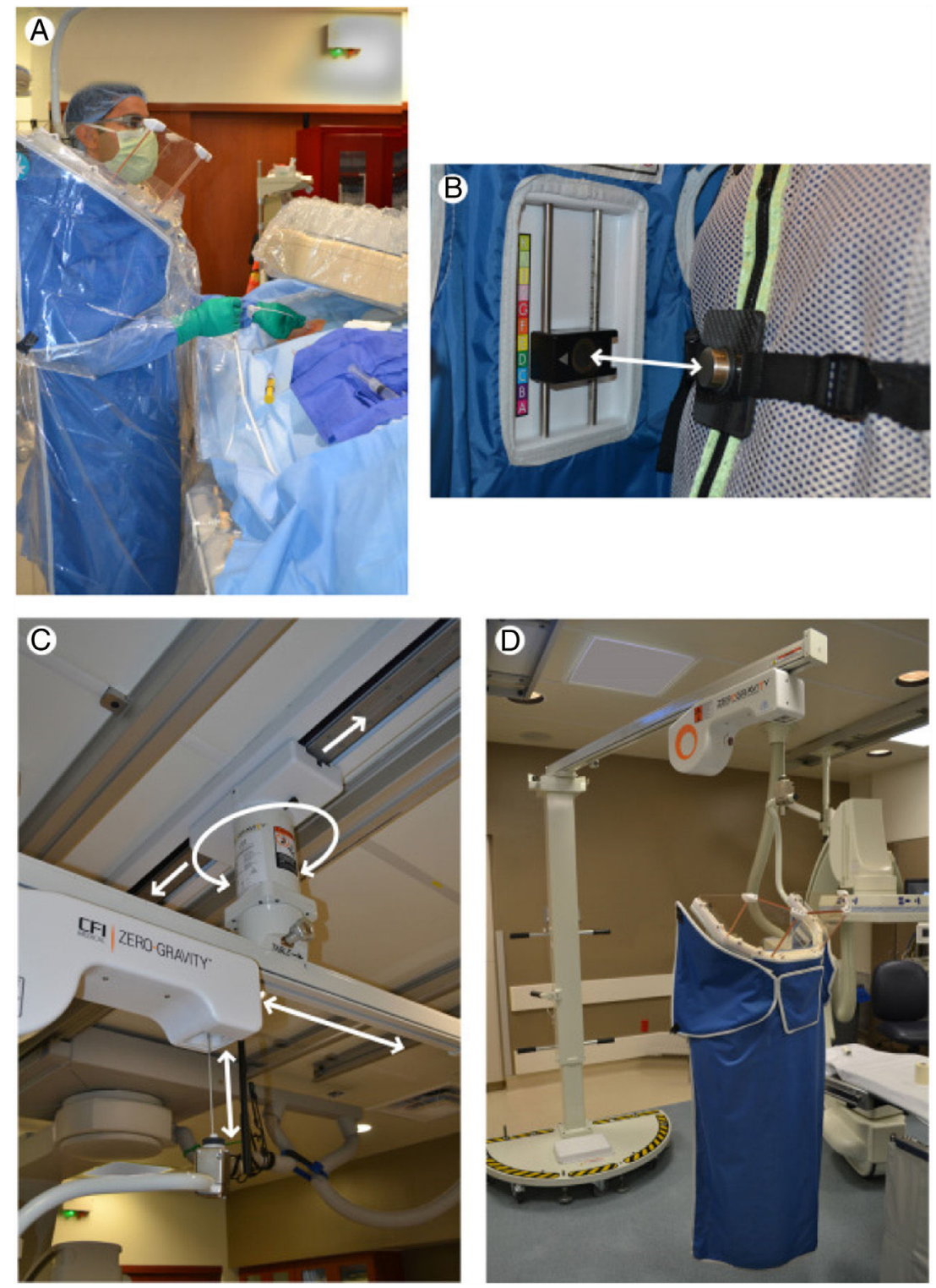

Figura 1 SPRPS (Zero-Gravity TM, productos Tidi, Fenton, MI). A) Chaleco protector. B) y C) Adaptador de chaleco. D) Sistema de suspensión de techo.

están incorporando alternativas sin plomo en el blindaje. No obstante, la literatura respecto a la atenuación proporcionada por el material compuesto más reciente es variada y requiere estudios adicionales centrados en la exposición en un amplio espectro $(0 \mathrm{a} 130 \mathrm{keV})^{18}$.

El SPRPS (Zero-Gravity TM, productos Tidi, Fenton, MI) (fig. 1) reemplaza el delantal con soporte del cuerpo por un sistema de protección personal más amplio que se suspende de la cabeza "sin peso" para que se mueva con el operador y mantenga una constante relación espacial optimizada con su cuerpo. Las principales ventajas de los SPRPS son la ausencia de cualquier peso soportado por el operador, la relación de atenuación más alta debido al uso de un delantal de plomo de $1 \mathrm{~mm}$, la cobertura del cuerpo expandida debido al escudo para la cabeza, los escudos de los brazos y la cobertura de la pierna inferior; la reducción de las brechas en la protección debido a la cobertura contigua desde la cabeza hasta la pantorrilla; el mantenimiento automático de la posición en la trayectoria de dispersión debido a la constante orientación al cuerpo a pesar del movimiento del operador; la ausencia de obstrucción al acceso del paciente; y la eliminación de las prendas y accesorios estándar. La principal desventaja es el alto costo (figs. 2 y 3 ).

El uso de blindaje complementario generalmente se recomienda cuando se emplean delantales de plomo convencionales. Los estándares aceptados incluyen una lámina flexible de listones de plomo (también conocido como blindaje debajo de la mesa), que cuelga de un lado de la mesa y cerca del piso, un blindaje lateral que se adhiere a la parte superior de la mesa y se extiende unos pocos centímetros hacia arriba, y un protector de vidrio de plomo o acrílico suspendido, móvil, que generalmente se apoya en el techo.

El protector debajo de la mesa se puede usar en la mayoría de las posiciones del paciente y los ángulos de los tubos 


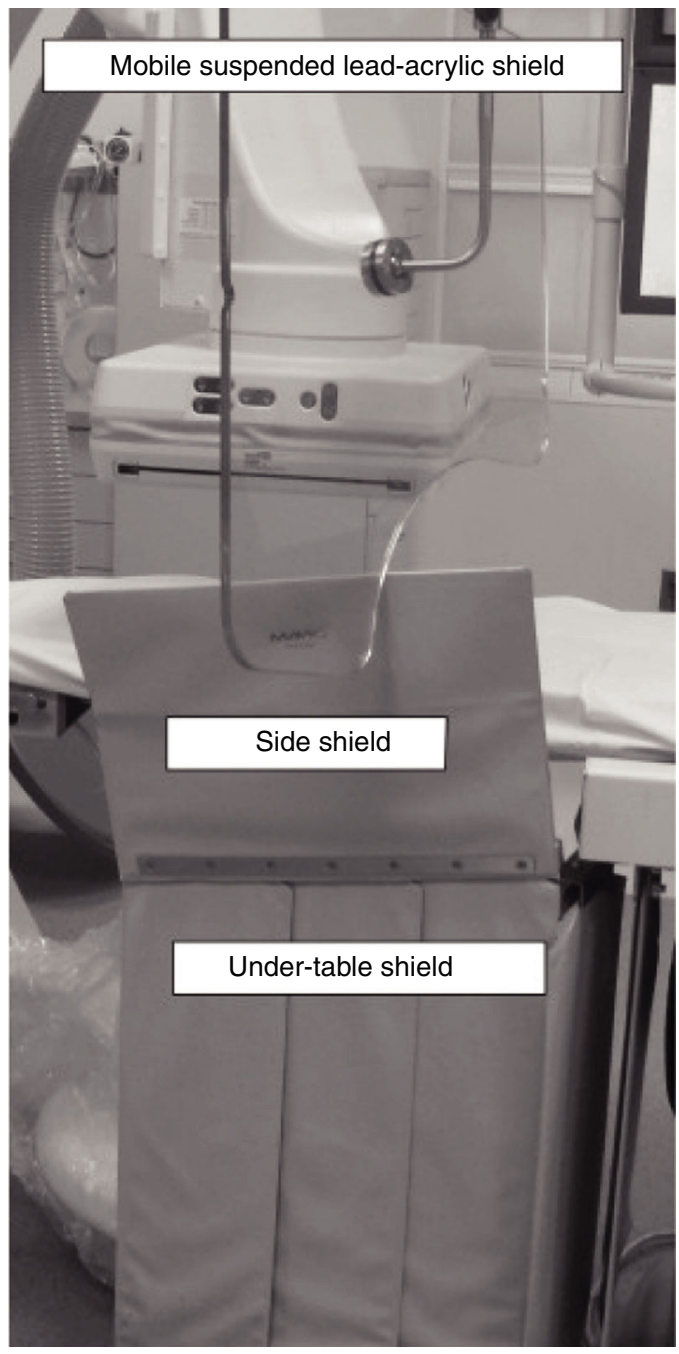

Figura 2 Blindaje complementario apoyado en el piso.

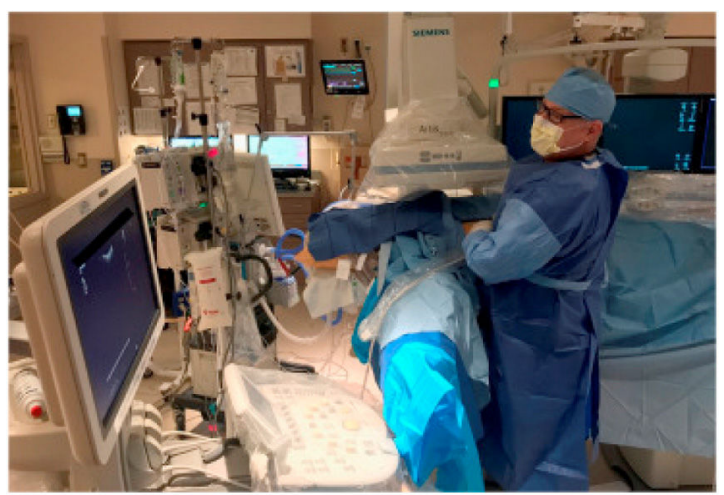

Figura 3 Posición ergonómica inadecuada del cardiólogo intervencionista.

con la necesidad de un reposicionamiento ocasional. El protector de la mesa lateral obstruye el esfuerzo de trabajo en algunos tipos de procedimientos y generalmente es removible. Debe ser removido para transferir al paciente y debe ser reemplazado por cada procedimiento donde no sea obstructivo. El protector móvil de acrílico de plomo se coloca en la posición adecuada antes y durante los procedimientos, pero requiere ajustes frecuentes por parte del operador, ya que los cambios se realizan en la posición de la mesa, el ángulo y la posición del tubo, la ubicación del cuerpo que se está fotografiando y las posiciones del operador y otros para mantener el efecto sheidling.

Los protectores móviles apoyados en el piso son una forma muy conveniente para reducir sustancialmente la exposición de todo el cuerpo al personal que no se comunica directamente con el paciente durante la preponderancia del tiempo de fluoroscopia. Las enfermeras circulan con frecuencia durante los procedimientos $y$, por tanto, deben usar delantales, pero pueden pasar parte del tiempo de fluoroscopia a una distancia del paciente y detrás de un protector móvil apoyado en el piso. Si se confirma por las bajas lecturas de la placa de sobre-plomo, esto puede permitir el uso de un delantal ligero, que no sería satisfactorio para el operador que pasa casi todo el tiempo de fluoroscopia en la zona "caliente".

Permanecer detrás de un escudo móvil apoyado en el piso sin un delantal puede ser seguro si tanto el escudo como la persona están colocados correctamente y no están en movimiento, y se los controla cuidadosamente con un dosímetro personal para garantizar la seguridad, bajo la guía del oficial de seguridad radiológica. Esto puede ser útil para un empleado con problemas músculo-esqueléticos limitantes. Estos escudos también pueden servir para la "fluoroscopia por TC" y pueden obviar la necesidad de un delantal de plomo si se colocan y se usan cuidadosamente con monitorización ${ }^{29}$.

Aunque se ha avanzado en la identificación de la ergonomía adecuada para el radiólogo de diagnóstico ${ }^{19}$, los cardiólogos intervencionistas pasan el tiempo como diagnosticadores y en procedimientos. Debido al uso de la fluoroscopia, la protección radiológica para el operador y el personal debe ser una prioridad absoluta. Se deben seguir las recomendaciones específicas para el diseño de la sala de angiografía, en particular respecto al blindaje contra la radiación. El blindaje a menudo crea desventajas ergonómicas para el médico operario. Se necesita una relación cercana entre los expertos en radiación calificados, los médicos y la administración para el diseño eficiente de la sala de radiología intervencionista ${ }^{20}$.

La altura de la mesa es un importante problema ergonómico para todos los procesadores. Un estudio de 2002 evaluó la posición correcta de la mesa durante los procedimientos. Como se mencionó anteriormente, tanto cirujanos laparoscopistas como cardiólogos intervencionistas usan puntos de entrada fijos para su equipo operativo, lo que sugiere fuertes paralelos entre ambas especialidades. Se encontró que la altura óptima de la superficie de operación se encuentra entre un factor de 0,7 a 0,8 de la altura del codo del cirujano $^{21}$.

A esta altura de la mesa, la articulación del codo está en una posición neutral durante el $90 \%$ del tiempo de operación, lo que permite que el bíceps braquial permanezca dentro de $15 \%$ de la actividad muscular máxima. En este estudio se encontró que la altura óptima de la mesa reduce las molestias en la espalda, los hombros y la muñeca durante la cirugía laparoscópica. Curiosamente, la altura de tabla óptima propuesta no fue una altura de tabla alcanzable en el estudio. Se necesitan trabajos adicionales para definir la altura óptima de la mesa para los cardiólogos intervencionistas. 
En cuanto a la posición del monitor, idealmente debe estar en el plano horizontal. El monitor de la pantalla debería colocarse directamente hacia adelante del operador y en línea con el eje del motor del antebrazo. Esto evitará la rotación axial de la columna vertebral. En el plano sagital, los monitores deben colocarse más abajo del nivel de los ojos para evitar la extensión del cuello. Los estudios han encontrado que la posición de visualización más cómoda es en una dirección descendente de aproximadamente 15 grados $^{22}$. Cualquier compañero, residente o tecnólogo que lo ayude debe colocarse de manera que no interrumpa el eje ojo-mano-objetivo del médico operario ${ }^{23}$.

Por otra parte, la ecografía es una herramienta útil para los radiólogos intervencionistas. De igual forma, hay varias recomendaciones útiles para la configuración ideal del aparato de ultrasonido ergonómico y la prevención de lesiones en la literatura sobre ultrasonido. Las recomendaciones específicas incluyen uso de iluminación indirecta (que se controla a través de un reóstato), minimización de la abducción del brazo y posicionamiento del monitor directamente frente al usuario para que el ecografista vea la parte superior de la pantalla sin torsión axial ${ }^{24}$. Estudios adicionales de la literatura ecográfica continúan recomendando el mantenimiento de la posición neutra del cuerpo para ayudar a evitar lesiones futuras.

\section{Recomendaciones para la prevención de lesiones}

Luego de una revisión cuidadosa de la literatura es evidente que la comunidad de cardiólogos intervencionistas carece de estándares de seguridad laboral cohesivos diseñados para minimizar las lesiones músculo-esqueléticas relacionadas con el trabajo. Las recomendaciones de sentido común respecto a las formas de minimizar las lesiones músculo-esqueléticas relacionadas con el trabajo a largo plazo incluyen las siguientes:

- Mantenga un buen estado físico general fuera del trabajo, con énfasis específico en la fuerza del cuerpo central.

- Practique una buena postura mientras opera, en posición vertical, sin inclinarse hacia adelante o hacia los lados, de modo que se evite la tensión en la espalda ${ }^{25}$.

- Ubique los monitores de manera que se evite torcer o extender el cuello directamente hacia adelante y aproximadamente 15 grados por debajo del nivel de los ojos.

- Use prendas de protección de ajuste apropiado con envoltura y diseño de dos piezas. Limite su uso a la sala de procedimientos de fluoroscopia y retírelos en todas las demás áreas de trabajo. Utilice un cinturón de soporte lumbar/abdominal. Descanse intermitentemente un pie en un bloque de 6 pulgadas durante los procedimientos largos. También puede ser útil caminar y estirar la espalda durante un caso largo ${ }^{25}$.

\section{Direcciones futuras}

El desarrollo de manipuladores remotos que utilizan robótica es una solución prometedora para los problemas ergonómicos que se originan en las prendas de protección. Otras especialidades quirúrgicas, como la urología, ya utilizan robots quirúrgicos. El sistema quirúrgico "da Vinci" se usa en una variedad creciente de indicaciones ${ }^{26}$. Otra posibilidad interesante incluye el uso de pantallas montadas en la cabeza. En un sistema de este tipo, la tomografía computarizada y las imágenes de rayos $X$ se proyectan para que el médico evite lesiones ergonómicas.

\section{Creative commons}

Creative Commons Reconocimiento-No Comercial-Sin Obra Derivada (CC BY-NC-ND).

\section{Conflicto de intereses}

Ninguno.

\section{Bibliografía}

1. Andersson GB. Epidemiologic aspects on low-back pain in industry. Spine (PhilaPa 1976). 1981;6:53-60.

2. Frymoyer JW, Pope MH, Clements JH, Wilder DG, MacPherson B, Ashikaga T. Risk factors in low-back pain. J Bone Joint Surg [Am]. 1983;65-A:213-8.

3. Pope MH, Frymoyer JW, Andersson G. Occupational low back pain. New York: Praeger; 1984. p. 101-54.

4. Snook SH. Low back pain in industry. En: White AA, Gordon SL, editores. Symposium on idiopathic low back pain. St. Louis: Mosby; 1982. p. 23-8.

5. Steinberg GG. Epidemiology of low back pain. En: Stanton-Hicks M, Boas R, editores. Chronic low back pain. New York: Raven; 1982. p. 1-13.

6. Svensson HO, Andersson GBJ. Low-back pain in 40- to 47-yearold men: work history and work environment factors. Spine. 1983;8:272-6.

7. Brown JR. Factors contributing to the development of low-back pain in industrial workers. J Am Ind Hyg Assoc. 1975;35:26-31.

8. Frymoyer JW, Pope MH, Costanza MC, Rosen JC, Goggin JE, Wilder DG. Epidemiologic studies of low-back pain. Spine. 1980;5:419-23.

9. Magora A. Investigation of the relation between low back pain and occupation: Medical history and symptoms. Scand J Rehabil Med. 1974;6:81-8.

10. Machan L. A web-based survey of neck and back pain amongst interventional radiologists. J Vasc Interv Radiol. 2001;12 suppl:S28.

11. Moore B, van Sonnenberg E, Casola G, Novelline RA. The relationship between back pain and lead apron use in radiologists. Am J Roentgenol. 1992;158:191-3.

12. Ross AM, Segal J, Borenstein D, Jenkins E, Cho S. Prevalence of spinal disc disease among interventional cardiologists. Am J Cardiol. 1997;79:68-70.

13. Goldstein JA, Balter S, Cowley M, Hodgson J, Klein LW. Occupational hazards of interventional cardiologists: Prevalence of orthopedic health problems in contemporary practice. Catheter Cardiovasc Interv. 2004;63:407-11.

14. Center for Diseases Control and Prevention, National Center for Health Statistics. National Health Interview Survey (NHIS) [Internet]. Washington: CDC, NCHS; 1997. [Acceso 1 Mar 2019]. Disponible en: http://www.cdc.gov/nchs/about/ major/nhis/quest_data_related_1997_forward.htm.

15. Schiller JS, Lucas JW, Peregoy JA. Summary health statistics for U.S. Adults: national health interview survey, 2011. Vital Health Stat 10. 2012:1-218.

16. Cremen SA, McNulty JP. The availability of appropriately fitting personal protective aprons and jackets for 
angiographic and interventional radiology personnel Radiography. 2014;20:126-30.

17. Akbar-Khanzadeh F, Bisesi MS, Rivas RD. Comfort of personal protective equipment. Appl Ergon. 1995;26:195-8.

18. Meisinger QC, Stahl CM, Andre MP, et al. Radiation protection for the fluoroscopy operator and staff. Am J Roentgenol. 2016;207:745-54, http://dx.doi.org/10.2214/AJR.16.16556.

19. Harisinghani MG, Blake MA, Saksena $M$, et al. Importance and effects of altered workplace ergonomics in modern radiology suites. Radiographics. 2004;24:615-27, http: //dx.doi.org/10.1148/rg.242035089.

20. Chalmers GJ. Structural shielding design for medical imaging x-ray facilities (NCRP Report No. 147). Phys Med Biol. 2005;50:4243-4, http://dx.doi.org/10.1088/ 0031-9155/50/17/B01.

21. van Veelen MA, Kazemier G, Koopman J, Goossens RH, Meijer DW. Assessment of the ergonomically optimal operating surface height for laparoscopic surgery. J Laparoendosc Adv Surg Tech A. 2005;12:47-52, http://dx.doi.org/10. 1089/109264202753486920.

22. Zehetner J, Kaltenbacher A, Wayand W, Shamiyeh A. Screen height as an ergonomic factor in laparoscopic surgery. Surg Endosc Other Interv Tech. 2006;20:139-41, http://dx.doi.org/10.1007/s00464-0050251-1.
23. Van Det MJ, Meijerink WJ, Hoff C, et al. Optimal ergonomics for laparoscopic surgery in minimally invasive surgery suites: A review and guidelines. Surg Endosc Other Interv Tech. 2009;23:1279-85, http://dx.doi.org/10. 1007/s00464-008-0148-x.

24. Baker JP, Coffin CT. The importance of an ergonomic workstation to practicing sonographers. J Ultrasound Med. 2013:1363-75, http://dx.doi.org/10.7863/ultra.32.8.1363.

25. Naito $\mathrm{N}$. Occupational back pain in the interventional radiologist. J Vasc Interv Radiol. 2005;16:312-4, http://dx.doi.org/ 10.1016/S1051-0443(05)70244-X.

26. Maeso S, Reza M, Mayol JA, Blasco JA, Guerra M, Andradas $E$, et al. Efficacy of the Da Vinci surgical system in abdominal surgery compared with that of laparoscopy: a systematic review and meta-analysis. Ann Surg. 2010;252:254-62, http://dx.doi.org/10.1097/SLA.0b013e3181e6239e.

27. Rothmore P. Lead aprons, radiographers, and discomfort: A pilot study. J Occup Health Saf Aust N Z. 2002;18:357-66.

28. Orme NM, Rihal CS, Gulati R, Holmes DR Jr, Lennon RJ, Lewis BR, et al. Occupational health hazards of working in the interventional laboratory: a multisite case control study of physicians and allied staff. J Am Coll Cardiol. 2015;65:820-6.

29. Rees CR, Duncan BWC. Get the lead off our backs! Tech Vasc Interv Radiol. 2018;21:7-15. 